\title{
AN OSCILLATOR MODEL FOR STELLAR VARIABILITY
}

\author{
T. NAKAHARA and Y. TANAKA \\ Faculty of Education, Jbaraki University, Bunkyo, Mito 310, Japan
}

\begin{abstract}
The dependence of coupling constants in a coupled oscillator model is examined with simplified methods. The Lyapunov exponents are preliminary introduced for the model. The behaviors of oscillator model are examined in a parameter plane. So-called the Arnold's tongues for phase-locking states are observed in fractal patterns.
\end{abstract}

\section{Introduction}

Two-mode coupling oscillator model for stellar variability has investigated in the view of nonlinear dynamics (Seya et al. 1989). The equations of coupled oscillator model are described as follows;

$$
\begin{aligned}
& \frac{d^{2} x_{1}}{d t^{2}}=-\sigma_{1}^{2} x_{1}+\sigma_{1}^{2}\left\{\left[(1 / 2) C_{111} x_{1}+C_{112} x_{2}\right] x_{1}\right. \\
& \left.+\varepsilon_{1}\left(1-\alpha_{1}^{2} x_{1}^{2}\right)\left(d x_{1} / d t\right)+(1 / 2) C_{122} x_{2}^{2}\right\}, \\
& \begin{aligned}
\frac{d^{2} x_{2}}{d t^{2}}=-\sigma_{2}^{2} x_{2}+\sigma_{2}^{2}\left\{\left[(1 / 2) C_{222} x_{2}+C_{212} x_{1}\right] x_{2}\right. \\
\left.+\varepsilon_{2}\left(1-\alpha_{2}^{2} x_{2}^{2}\right)\left(d x_{2} / d t\right)+(1 / 2) C_{211} x_{1}^{2}\right\},
\end{aligned}
\end{aligned}
$$

where the coupling constants $C_{i j k}$ are evaluated by Takeuti (1985) for a classical cepheid model. It is featured that the van der Pol's damping term is introduced. Seya et al. (1989) show the complicated behaviors of Eq. (1) as the change of coefficients in damping terms. The behaviors seem to be phase-locking, quasi-periodicity or chaos. Using these equations, we examine the dependence of behaviors on coupling constants by rather simple manner. We simplify the constants as $C_{i j k}=C_{i}$ for all $j, k$. The evaluated values of $C_{1 j k}$ and $C_{2 j k}$ by Takeuti (1985) equal $2 \sim 4$ and 6 , respectively. Thus the value of $C_{1}$ is varied from 2 to 6 and that of $C_{2}$ is fixed at 6.0 . It should be worthwhile to research the tendency of solutions for wide values of $C_{1}$ and $C_{2}$. The set of angular frequencies is chosen as $\sigma_{1}^{2}=1.0$ and $\sigma_{1}^{2}=1.9$.

\section{Results}

First, we follow the results by Tanaka et al. (1991a) that the Lyapunov exponents are preliminary introduced in order to distinguish the solutions of Eq. (1). The computed results is shown in Table I. In these case, all $\lambda_{1}$ is nearly zero and $\lambda_{2}$ is zero and negative, which mean that quasi-periodicity or phase-locking occur. We also examined time developments and projected orbits on a phase plane for the same parameter and confirmed the state of 
solutions in the Table. We should note that the other phase-locking states appear as the values of coupling constants and coefficients of damping terms are varied. The frequency ratios cover from $2 / 3$ to $1 / 1$ as Farey's series, while indistinguishable (quasi-periodic, chaotic and convergent) and divergence cases are also observed. These have been observed as the ratio of angular frequency is changed (Tanaka et al. 1990).

TABLE I

The samples of the Lyapunov exponents and the period ratios. The coupling constants and parameters of nonlinear damping term are also given. $\sigma_{1}^{2}=1.0, \sigma_{2}^{2}=1.9$, $\varepsilon_{1}=0.1$. Q-P means the quasi-periodicity.

\begin{tabular}{|c|c|c|c|c|c|c|c|c|c|}
\hline \multicolumn{5}{|c|}{ Parameters } & \multicolumn{4}{|c|}{ Lyapunov exponents } & \multirow[b]{2}{*}{$P_{2} / P_{1}$} \\
\hline$C_{1}$ & $C_{2}$ & $\varepsilon_{2}$ & $\alpha_{1}^{2}$ & $\alpha_{2}^{2}$ & $\lambda_{1}$ & $\lambda_{2}$ & $\lambda_{3}$ & $\lambda_{4}$ & \\
\hline 6.0 & 6.0 & 0.3 & 1600 & 1600 & 0.000 & 0.000 & -0.148 & -0.845 & Q-P \\
\hline 6.0 & 6.0 & 0.3 & 1600 & 800 & 0.001 & -0.016 & -0.141 & -0.872 & $7 / 10$ \\
\hline 6.0 & 6.0 & 0.4 & 1600 & 800 & 0.000 & -0.021 & -0.149 & -1.195 & $5 / 7$ \\
\hline 6.0 & 6.0 & 0.3 & 1600 & 2400 & 0.000 & -0.031 & -0.113 & -0.844 & $3 / 4$ \\
\hline 2.0 & 6.0 & 0.1 & 800 & 800 & 0.000 & -0.063 & -0.064 & -0.329 & $4 / 5$ \\
\hline
\end{tabular}

Next, the behaviors of coupled oscillator are studied by Tanaka et al. $(1991 \mathrm{~b})$ on the parameters plane, $\left(C_{1}, C_{2}\right)$ where we shall see fractal patterns. Numerical integration are carried out by the mixed Euler and Heun method with a personal computers. The first diagram is divided into two regions, divergent or non-divergent. The divergent region (D) is defined by $A=x_{1}^{2}+x_{2}^{2}>10$. When $\mathrm{A}$ becomes greater than 10 , the pixel at $\left(C_{1}, C_{2}\right)$ is colored by the number of iteration. When $A$ keeps to be small after the 500 iteration, the set of $\left(C_{1}, C_{2}\right)$ is seemed as non-divergent region (ND) and colored black. In the diagram, $\mathrm{D}$ region is patterned by complex stripes. When $\alpha_{1}^{2}$ becomes large and $\alpha_{2}^{2}$ small, ND region tends to expand. They also illustrate the structure of ND region. Although phase-locking, quasiperiodicity and chaos are included in ND region, they only distinguish the phase-locking and color according to counted ratios. We seem that these regions are so-called the Arnold's tongues, which appear in the circle map. We can understand that the coupled oscillator model behaves as a simple sine map which is the simplified form of forced oscillator.

\section{Concluding Remarks}

We have shown the model coupling oscillator models for stellar variability by using simplified sets of constants. The route to chaos in the coupled oscillator are period doubling and quasi-periodicity which depends upon the control parameters. 


\section{References}

Seya, K., Tanaka, Y., and Takeuti, M.: 1990, Publications of the ASJ 42, 405.

Takeuti, M.: 1985, Astrophysics and Space Science 109, 99.

Tanaka, Y., Nakahara, T., and Yokata, Y.: 1991a, Bull. Fac. Educ., Ibaraki Univ., in press

Tanaka, Y., Ogura, S., and Sekino. T.: 1991b, Bull. Fac. Educ., Ibaraki Univ., in press.

Tanaka, Y., Seya, K., and Takeuti, M.: 1990, in Confrontations between Stellar Pulsation and Evolution, eds. C. Cacciari and G. Clementini (Astronomical Society of Pacific), 145 . 